\title{
Reliability of the diagnosis of a first seizure
}

\author{
C.A. van Donselaar \\ Dept. Neurology, St. Clara Ziekenhuis and University Hospital Rotterdam Dijkzigt, Rotterdam. The Netherlands \\ (Received 22 December, 1992) \\ (Accepted 11 January, 1993)
}

\section{Dear Sir,}

In their article "Syncope or seizure? A matter of opinion" Hoefnagels et al. [1] conclude that "the diagnosis of a seizure after a single event is often too unreliable to justify early treatment". In my view, the data presented by the authors do not support this conclusion and might even lead to an opposite judgment.

The authors used kappa-statistics to assess the interobserver variability. These statistics are difficult to interpret since they depend on the number of categories used. the prevalence of the disease, etc. The kappa for the two neurologists using three categories (seizure, syncope, don't know) was 0.40 , which is considered to be poor. If they would have used two categories instead (seizure yes/ no) the kappa would have been 0.65 which is valued by the authors as "substantial" and hence reverse the conclusion. The raw data for the other participants were not presented.

The two neurologists disagreed most often on the cate- gories "don't know" versus syncope and that is far less important from a clinical point of view than disagreement on the question seizure yes or no. Another solution would have been to use weighted kappa-statistics to take into consideration the clinical value of the disagreement [2].

In my view, the conclusion should have been that the reliability of the diagnosis of a seizure between neurologists is substantial. Whether this justifies early treatment is a different question.

\section{References}

1 Hoefnagels, W.A.J., Padberg. G.W.. Overweg, J. and Roos, R.A.C. (1992) Syncope or seizure? A matter of opinion. Clin. Neurol. Neurosurg., 94: 153-156.

2 Cohen, J. (1988) Weighted kappa: nominal scale agreement with provision for scaled disagreement or partial credit. Psychol. Bull., 29: 787-793. 